\title{
Prospective Study of Rapid Relief Provided by C1 Esterase Inhibitor in Emergency Treatment of Acute Laryngeal Attacks in Hereditary Angioedema
}

\author{
Timothy J. Craig • Richard L. Wasserman • Robyn J. Levy • Againdra K. Bewtra • \\ Lynda Schneider • Flint Packer • William H. Yang • Heinz-Otto Keinecke • \\ Peter C. Kiessling
}

Received: 13 April 2010 / Accepted: 30 June 2010 / Published online: 16 July 2010

(C) The Author(s) 2010. This article is published with open access at Springerlink.com

\begin{abstract}
Introduction Hereditary angioedema (HAE) is a rare disorder characterized by $\mathrm{C} 1$ esterase inhibitor (C1-INH) deficiency, resulting in periodic attacks of acute edema that can be life-threatening if they occur in the laryngeal region. We assessed the efficacy of $\mathrm{C} 1-\mathrm{INH}$ concentrate in the emergency treatment of rarely occurring acute laryngeal HAE attacks in a prospective, open-label clinical study. Methods Acute laryngeal attacks were each treated with $\mathrm{C} 1-\mathrm{INH}$ concentrate (Berinert) at a single dose of $20 \mathrm{U} / \mathrm{kg}$ body weight. Efficacy endpoints included time to onset of symptom relief and time to complete resolution of all symptoms, each based on the patient's assessment.

Results All 39 laryngeal attacks in 16 patients were treated
\end{abstract}

Financial Support This study was funded by CSL Behring GmbH Marburg, Germany.

T. J. Craig $(\bowtie)$

Asthma and Respiratory Research, Penn State University,

500 University Drive,

Hershey, PA 17033, USA

e-mail: tcraig@hmc.psu.edu

R. L. Wasserman

Dallas Allergy Immunology,

Dallas, TX, USA

R. J. Levy

Family Allergy and Asthma Center,

Atlanta, GA, USA

\section{A. K. Bewtra}

Creighton University School of Medicine,

Omaha, NE, USA

\section{Schneider}

Children's Hospital,

Boston, MA, USA successfully. The median time to onset of symptom relief was $15 \mathrm{~min}$. The median time to complete resolution of all symptoms was $8.25 \mathrm{~h}$. No treatment-related serious adverse events occurred, and the treatment was well tolerated. The administration of $\mathrm{C} 1-\mathrm{INH}$ concentrate was not associated with any viral infections.

Conclusion C1-INH concentrate is an effective and safe emergency treatment for providing reliable and rapid relief from the potentially life-threatening symptoms of laryngeal HAE attacks.

Keywords C1 inhibitor concentrate. hereditary angioedema $\cdot$ laryngeal attack · C1 inhibitor replacement therapy

F. Packer

Family First Medical Center,

Idaho Falls, ID, USA

W. H. Yang

Allergy \& Asthma Research Centre,

Ottawa, Canada

H.-O. Keinecke

Accovion $\mathrm{GmbH}$,

Marburg, Germany

P. C. Kiessling

CSL Behring GmbH,

Marburg, Germany 


\section{Abbreviations used \\ C1-INH $\quad \mathrm{C} 1$ esterase inhibitor \\ HAE Hereditary angioedema \\ I.M.P.A.C.T International Multicenter Prospective Angioedema C1-INH Trial}

\section{Introduction}

Hereditary angioedema (HAE) is a rare disease in which an inherited functional deficiency of $\mathrm{C} 1$ esterase inhibitor (C1INH) is clinically characterized by unpredictable episodes of edema at various body locations [1,2]. C1-INH is an important control protein of the complement and kallikreinkinin cascade systems [3]. During HAE attacks, these plasma proteolytic cascades are activated and, due to either the complete lack of C1-INH (type I HAE) or the expression of dysfunctional C1-INH (type II HAE), result in enhanced vascular permeability, leading to local subcutaneous or submucous swellings. The most frequent symptoms of HAE are acute episodes of skin swellings or abdominal pain that can be inconvenient and painful but which are not life-threatening. In contrast, the rare (approximately $1 \%$ of HAE attacks [4]) but potentially life-threatening episodes of laryngeal edema are associated with a risk of asphyxiation, which is the most important symptom of concern for patients with HAE [5]. Clinical manifestations of laryngeal attacks include hoarseness, stridor, dyspnea, the feeling of having a lump in the larynx, dysphagia, and voice change $[4,6]$.

The first-line therapy for acute HAE attacks is replacement therapy with $\mathrm{C} 1-\mathrm{INH}$ concentrate as recommended in the 2007 International Consensus Algorithm for the Management of HAE [7]. Before the availability of effective treatments, up to $25 \%$ to $30 \%$ of patients with HAE died from laryngeal edema [8]. An even higher mortality of approximately $50 \%$ has been reported for patients with undiagnosed HAE [9]. Since the introduction of C1-INH concentrate in several countries, the rate of mortality has decreased dramatically, but patients with HAE still die from laryngeal attacks that are not treated with $\mathrm{C} 1$ INH concentrate $[10,11]$. Due to the danger of asphyxiation, it is vital that acute attacks of laryngeal edema are treated effectively before reaching their maximum development. During this time, emergency intervention procedures include the administration of C1-INH concentrate, the administration of oxygen, intubation, and tracheotomy [5].

The efficacy and safety of C1-INH concentrate have been demonstrated in clinical use for more than 30 years, involving over 400,000 treatments in patients with HAE; this evidence is based mainly upon the treatment of acute abdominal and facial attacks using a fixed dose of C1-INH concentrate $[2,12-15]$. Information on the efficacy of $\mathrm{C} 1$ INH concentrate in the emergency treatment of laryngeal edema is limited [16].

Here, we report on data obtained from 39 laryngeal attacks in 16 patients with HAE that were treated with $\mathrm{C} 1$ INH concentrate (Berinert; CSL Behring, Marburg, Germany) in a prospective, open-label clinical study (International Multicenter Prospective Angioedema, C1INH Trial 2 [I.M.P.A.C.T.2]). This highly purified C1-INH concentrate is produced from human plasma obtained in the USA. It is rendered free of detectable virus by two independent steps of virus inactivation and removal [17]. The I.M.P.A.C.T.2 study is an extension of the placebocontrolled I.M.P.A.C.T.1 study that had previously established $20 \mathrm{U} / \mathrm{kg}$ as an effective dose of C1-INH concentrate in the treatment of acute HAE attacks [15]. While only single abdominal and facial HAE attacks were treated in I. M.P.A.C.T.1, repeated attacks in all body locations were treated in I.M.P.A.C.T.2. Here, we analyze the treatment of the laryngeal attacks that occurred in I.M.P.A.C.T.2. Our focus is to evaluate whether C1-INH concentrate given at the weight-based dose of $20 \mathrm{U} / \mathrm{kg}$, which had demonstrated efficacy compared to placebo against abdominal and facial attacks in I.M.P.A.C.T.1, is also as effective at providing rapid relief from the symptoms of laryngeal edema. Due to the life-threatening nature of laryngeal attacks and the evidence from retrospective reports for the efficacy of $\mathrm{C} 1$ INH concentrate in the treatment of laryngeal attacks, a placebo-controlled investigation of laryngeal attacks was not considered ethical.

\section{Methods}

\section{Study Design and Setting}

Laryngeal attacks were evaluated in this prospective, openlabel, uncontrolled extension study (I.M.P.A.C.T.2) in patients who had participated in the preceding I.M.P.A.C. T.1 study [15] at seven centers in North America. The data were obtained between 31 August 2005 and 24 August 2009.

Patients were treated with C1-INH concentrate $(50 \mathrm{U} / \mathrm{mL}$ after reconstitution) at a single intravenous dose of $20 \mathrm{U} / \mathrm{kg}$ body weight $(4 \mathrm{~mL} / \mathrm{min})$ for each attack evaluated at the study center, irrespective of the intensity of the attack. After treatment, patients remained at the study center and were observed until they reported onset of symptom relief. If a patient was discharged before complete resolution of an attack, a diary card was issued for the patient to document the time of complete resolution as well as any concomitant medication taken. In addition, the patients documented any adverse events experienced within 7 to 9 days after 
treatment of the attack. An HAE attack was only to be reported as an adverse event if the symptoms of a treated attack had worsened; new attacks were not to be reported as adverse events.

The study protocol was approved by the Institutional Review Board at each participating center or by a central Institutional Review Board. Written informed consent was obtained from each patient or, if the patient was a minor, from a legally acceptable representative. Assents were also obtained when appropriate. An independent data and safety monitoring board monitored the safety of the study.

\section{Selection of Participants}

Patients were eligible for treatment if they had previously been enrolled in the I.M.P.A.C.T.1 study, irrespective of their treatment in the study. The total sample size in this extension study was therefore limited by the number of patients who had been screened and found eligible for participation in I.M.P.A.C.T.1.

Other inclusion criteria included being at least 6 years of age with laboratory and historically confirmed $\mathrm{C} 1-\mathrm{INH}$ deficiency (type I or II HAE) and having a documented history of abdominal or facial attacks. Relevant exclusion criteria included a history of hypersensitivity to $\mathrm{C} 1-\mathrm{INH}$ concentrate, acquired angioedema due to $\mathrm{C} 1$-INH deficiency, all other types of angioedema not associated with $\mathrm{C} 1$ INH deficiency, use of any C1-INH concentrate within $24 \mathrm{~h}$ before the start of treatment in I.M.P.A.C.T.2, and use of fresh frozen plasma or native plasma within 7 days before the start of treatment.

\section{Methods of Measurement}

The primary endpoint was the time from start of treatment to onset of symptom relief, as determined by the patient's responses to a standard question posed at predetermined intervals for up to $24 \mathrm{~h}$ after the start of treatment. This type of endpoint has been widely used in the evaluation of HAE therapies [18-20]. In addition, for the preceding I.M.P.A.C. T.1 study, we retrospectively validated this primary endpoint by correlation with the course of the associated HAE symptoms (data on file). Secondary endpoints included time to complete resolution of all symptoms (patient's assessment), proportion of patients with at least one attack with onset of relief exceeding $4 \mathrm{~h}$, and proportion of attacks with a time to onset of relief exceeding $4 \mathrm{~h}$.

Adverse events and vital signs (systolic and diastolic blood pressure, pulse rate, respiratory rate, and body temperature) were recorded at appropriate times throughout the study. A viral safety assessment was performed before, and 7 to 9 days (parvovirus B19) or 12 weeks (human immunodeficiency virus type 1 and 2 , and hepatitis $\mathrm{A}, \mathrm{B}$, and $\mathrm{C}$ virus) after, treatment of the first attack in I.M.P.A.C.T.2.

Reflecting clinical practice, there were no restrictions for the time between the onset of an attack and the start of treatment, or for the use of prior or concomitant medications, including rescue medications such as analgesics and anti-emetics.

\section{Data Analysis}

Efficacy analyses were based on the intention-to-treat principle and included all patients and attacks treated with C1-INH concentrate. Descriptive statistics were calculated for all efficacy outcomes on a by-patient and by-attack basis, and confidence intervals were calculated for the median time to onset of symptom relief and the median time to complete resolution of all symptoms. For the bypatient analyses, the mean times for all attacks in a given patient were used. Missing values for time to onset of symptom relief were conservatively imputed as the maximum time to start of relief for the subject involved or, if no other time to onset of relief was available for the subject, as the time to complete resolution of all symptoms.

All patients who had received any treatment with $\mathrm{C} 1$ INH concentrate were included in the safety analysis. Adverse events were coded according to the Medical Dictionary for Regulatory Affairs (version 12.0), and incidence rates were calculated for adverse event preferred terms. No routine laboratory safety data were collected. Data for vital signs and viral safety were analyzed descriptively.

All data were analyzed using SAS version 9.1 (SAS Institute Inc., Cary, NC, USA).

\section{Results}

\section{Characteristics of Patients}

Each of the 39 laryngeal attacks occurring in 16 patients was treated with $\mathrm{C} 1-\mathrm{INH}$ concentrate. The median dose administered was $20 \mathrm{U} / \mathrm{kg}$ (range, 18-20 U/kg). The number of laryngeal attacks treated per patient ranged between one and eight, with seven patients treated for more than one laryngeal attack. A total of 14 patients were female, and two were male (Table I). The mean age was 32 years. All patients but two had type I HAE. The median time from the estimated start of the laryngeal HAE attack until the start of treatment was $2.8 \mathrm{~h}$ (range, 1-95 h). Of the 39 laryngeal attacks treated, 11 were mild in intensity, 16 were moderate, and 12 were severe.

Apart from systemic antihistamines, diuretics, and sex hormones taken by two patients each (one patient took 
Table I Baseline Characteristics of Patients Treated for Laryngeal HAE Attacks

\begin{tabular}{ll}
\hline Characteristic & Intention-to-treat population $(N=16)$ \\
\hline Sex, $n(\%)$ & $14(87.5)$ \\
Female & $2(12.5)$ \\
Male & \\
Age (years) & $32.3(12.49)$ \\
Mean (SD) & $13-53$ \\
Range & \\
Race, $n(\%)$ & $14(87.5)$ \\
Caucasian & $2(12.5)$ \\
Black & \\
Body mass index (kg/m $\left.{ }^{2}\right)$ & $26.6(4.12)$ \\
Mean (SD) & $22-37$ \\
Range & $n(\%)$ \\
Primary disease (type of HAE) & $14(87.5)$ \\
Type I & $2(12.5)$ \\
Type II &
\end{tabular}

$H A E$ hereditary angioedema, $N$ total number of patients with laryngeal attacks, $n$ number of patients with available data, $S D$ standard deviation

danazol), each of 13 classes of concomitant medication recorded at the time of C1-INH treatment was taken by no more than one patient each.

\section{Efficacy Outcomes}

The median time between the start of treatment and the onset of symptom relief was $0.25 \mathrm{~h}$ for the 39 laryngeal attacks analyzed (Table II). All attacks were treated successfully. The median time to onset of relief for individual mean values per patient was $0.44 \mathrm{~h}$. Within $1 \mathrm{~h}$ of the start of treatment, onset of relief had been reported in at least $95 \%$ of all attacks, and the time to onset of relief was $\leq 0.75 \mathrm{~h}$ in at least $85 \%$ of patients (Fig. 1).

The median time from start of treatment to complete resolution of all symptoms was $8.25 \mathrm{~h}$ when analyzed by attack and $5.87 \mathrm{~h}$ when analyzed as the mean value per patient (Table II). The time to complete resolution of all HAE symptoms was less than $16 \mathrm{~h}$ in $75 \%$ of patients and less than $24 \mathrm{~h}$ in $74 \%$ of attacks (Fig. 2). The onset of symptom relief occurred within $4 \mathrm{~h}$ after the start of treatment in all patients; the maximum time between the start of treatment and the onset of symptom relief was $1.3 \mathrm{~h}$.

The intra-subject variability in the time to onset of symptom relief for the treatment of successive laryngeal attacks was low in the seven patients who were treated for more than one laryngeal attack. The maximum variability occurred in one subject treated for eight attacks, with the time to onset of relief ranging from 0.12 to $1.0 \mathrm{~h}$.

\section{Safety and Tolerability}

Safety was evaluated in all 16 patients treated with C1-INH concentrate. There were no deaths. Only 2 of the 16 patients experienced an adverse event. None of the adverse events led to discontinuation of treatment. One patient experienced a serious adverse event of HAE, which was not related to the treatment. The attack resolved without sequelae after $4.4 \mathrm{~h}$. Contrary to the intended study design, this new attack was reported by the study center as an adverse event. The other patient had an adverse event of influenza-like illness, which was assessed by the investigator as being possibly related to the treatment. This adverse event resolved within 1 day without sequelae.

No consistent or clinically relevant changes in vital signs occurred during the study. None of the subjects tested positive for markers of human immunodeficiency virus, hepatitis virus, or parvovirus B19 when tested after the first administration of $\mathrm{C} 1-\mathrm{INH}$ concentrate.

\section{Discussion}

C1-INH concentrate is the recommended first-line treatment for HAE attacks at all body locations [7]. Because
Table II Analysis of Time to Onset of Symptom Relief and Time to Complete Resolution of all Symptoms in the Intentionto-Treat Population

$N$ number of patients/attacks with available data, $C I$ confidence interval, $S D$ standard deviation

${ }^{a}$ Analysis of mean time for all attacks per patient

\begin{tabular}{lll}
\hline Statistics & \multicolumn{2}{l}{ Time $(\mathrm{h})$} \\
\cline { 2 - 3 } & Patients $^{\mathrm{a}}(N=16)$ & Attacks $(N=39)$ \\
\hline Time to onset of symptom relief_primary efficacy & outcome & \\
Mean (SD) & $0.53(0.323)$ & $0.42(0.291)$ \\
Median (range) & $0.44(0.2-1.3)$ & $0.25(0.1-1.3)$ \\
Two-sided 95\% CI for median & {$[0.32 ; 0.72]$} & {$[0.25 ; 0.50]$} \\
Time to complete resolution of all symptoms & & $13.87(13.624)$ \\
Mean (SD) & $11.41(13.384)$ & $8.25(0.6-48.9)$ \\
Median (range) & $5.87(0.6-48.3)$ & {$[4.10 ; 21.50]$} \\
Two-sided 95\% CI for median & {$[2.05 ; 18.26]$} & \\
\hline
\end{tabular}


laryngeal attacks are potentially life-threatening, and because the medical community generally lacks knowledge about this rare disorder, it is recommended that patients have an emergency supply of C1-INH concentrate at home or with them when they travel. However, C1-INH concentrate has yet to be licensed in some countries for the treatment of HAE attacks. Other treatment options, including fresh frozen plasma and/or epsilon-amino-caproic acid, are variable in their efficacy and can be associated with significant inherent side effects [21]. Therapeutic options that are effective for histamine-induced angioedema, such as corticosteroids and antihistamines, are not effective in the treatment of HAE attacks [8].

Although there is now more than 20 years of clinical experience with $\mathrm{C} 1-\mathrm{INH}$ concentrate in the treatment of acute HAE attacks [2], information on its efficacy in the emergency treatment of laryngeal edema is limited [16]. Furthermore, almost all of the clinical experience with C1INH concentrate has been obtained using fixed dosing with 500 or $1,000 \mathrm{U}$. Until recently, few data had been published from randomized, controlled studies to demonstrate the efficacy of C1-INH concentrate compared to placebo. Where such studies had been conducted, only low numbers of patients were analyzed [18, 20]. This deficit was addressed by the recent I.M.P.A.C.T.1 study, which demonstrated the statistically significant efficacy of a weight-based dose of $\mathrm{C} 1$-INH concentrate $(20 \mathrm{U} / \mathrm{kg})$ compared to placebo in the treatment of abdominal and facial HAE attacks in 124 patients [15]. Laryngeal attacks were not investigated in I.M.P.A.C.T.1 due to ethical concerns about treating this potentially life-threatening type of attack with placebo.

Against this background, our current study used an open-label setting to assess whether the beneficial efficacy

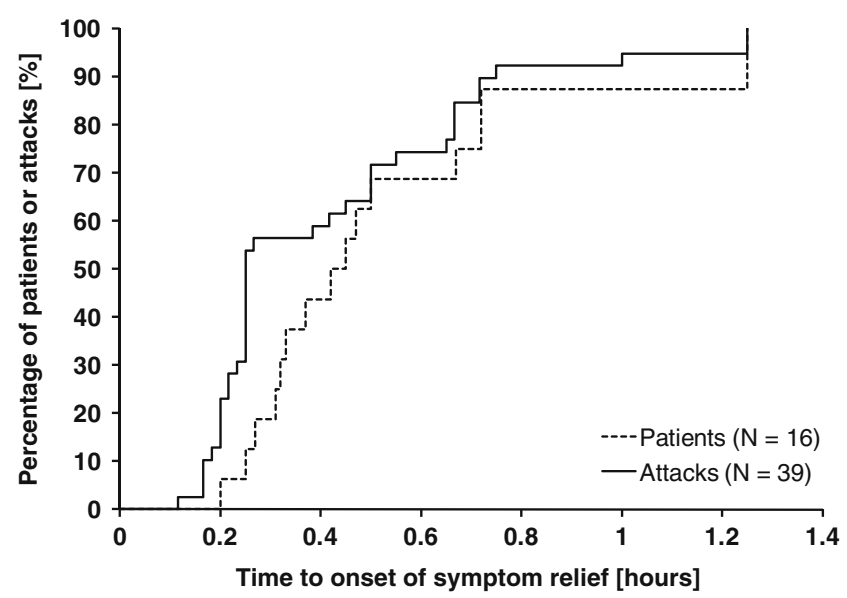

Fig. 1 Kaplan-Meier curves for time to onset of symptom relief, as determined by the patient's assessment. The curves show by-patient and by-attack analyses in both cases in the intention-to-treat population

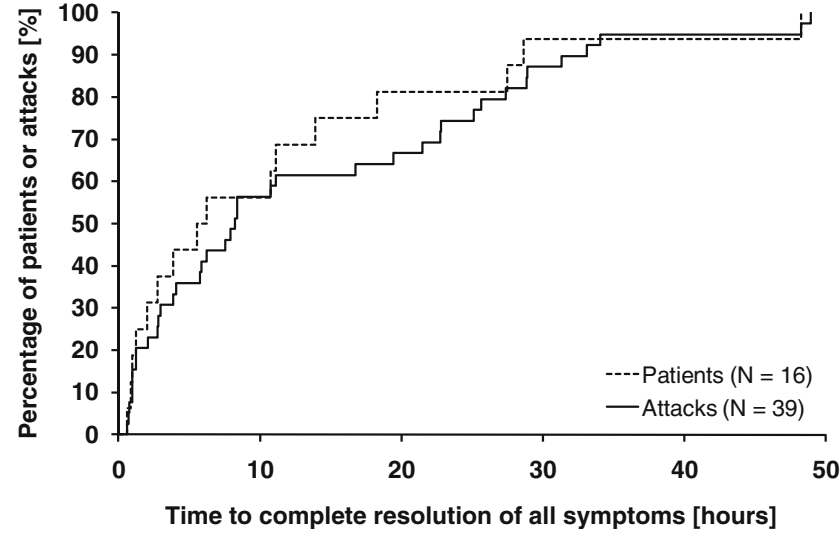

Fig. 2 Kaplan-Meier curves for time to complete resolution of all HAE symptoms, as determined by the patient's assessment. The curves show by-patient and by-attack analyses in both cases in the intention-to-treat population

of the $20 \mathrm{U} / \mathrm{kg}$ dose of C1-INH concentrate seen in the placebo-controlled I.M.P.A.C.T.1 study is also applicable to the treatment of laryngeal attacks. We prospectively investigated the efficacy of C1-INH concentrate in the emergency treatment of 39 acute laryngeal attacks in 16 patients with HAE. To our knowledge, this is the largest number of laryngeal attacks that have been investigated in a prospective study. The primary efficacy endpoint indicated that $\mathrm{C} 1-\mathrm{INH}$ concentrate given at a single dose of $20 \mathrm{U} / \mathrm{kg}$ induced a rapid onset of relief from the symptoms of the laryngeal attacks. The median time to onset of relief reported by the patients was $15 \mathrm{~min}$, thereby substantially reducing the risk of asphyxiation. In retrospective reports of laryngeal attacks treated with the same C1-INH concentrate at fixed doses of up to $1,000 \mathrm{U}$, the average time to onset of symptom relief was 15 to $42 \min [5,16,22]$. Similarly, for laryngeal attacks treated with a different $\mathrm{C} 1-\mathrm{INH}$ concentrate at doses of $25 \mathrm{U} / \mathrm{kg}$ or up to $2,000 \mathrm{U}$, the time to onset of symptom relief ranged from 20 to $60 \mathrm{~min}$ [20, 23].

Untreated laryngeal attacks can progress for 8 to $12 \mathrm{~h}$, after which the symptoms persist for 12 to $24 \mathrm{~h}$ and slowly subside thereafter, with a mean overall duration of $103 \mathrm{~h}$ having been reported for 342 untreated attacks [5, 24]. In the one controlled study in which laryngeal attacks were treated with placebo (four attacks in four patients), the mean time between treatment and onset of relief was $8.5 \mathrm{~h}$ [20]. The symptoms of laryngeal attacks treated with $\mathrm{C} 1-$ INH concentrate in our study started to subside rapidly by 6 to $78 \mathrm{~min}$ after treatment and were relatively short in duration (complete resolution after a mean of $13.9 \mathrm{~h}$ after treatment [median of $8.3 \mathrm{~h}$ ]). Thus, despite the unavoidable limitation of our study being uncontrolled, the comparison with these historical data for untreated or placebo-treated attacks nevertheless indicate the efficacy of C1-INH at the recommended dose of $20 \mathrm{U} / \mathrm{kg}$ in the treatment of laryngeal 
edema attacks. There was no evidence in our study for rebound edema after treatment of laryngeal attacks with C1INH concentrate, as indicated by no new attacks having occurred before complete resolution of the previous attack [15]. Compared to acute HAE attacks at other body locations, laryngeal attacks show the most rapid response to infusion of C1-INH concentrate [22], which was supported by our results for abdominal, facial, and peripheral attacks treated with the same weight-based dose of C1-INH concentrate in the I.M.P.A.C.T.2 study (median time to onset of symptom relief was 20 min for abdominal attacks, 28 min for facial attacks, and 31 min for peripheral attacks) (data on file).

Rare laryngeal attacks are experienced by approximately $50 \%$ of HAE patients at least once in their lifetime and represent a continuous threat to these patients because they mostly occur spontaneously and without warning [4, 5, 25]. An increased risk of developing laryngeal edema has been associated with the following factors: age between 11 and 45 years, general anesthesia with intubation, history of laryngeal edema, dental surgery, and facial edema. Although the first clinical symptoms of HAE are generally abdominal or facial attacks, and laryngeal attacks occur later in life [5], the first clinical symptom of HAE can nevertheless be a lifethreatening laryngeal attack, as demonstrated by the case of a 9-year-old boy who died from a laryngeal attack that was the first clinical sign of his known C1-INH deficiency [10]. The rapid onset of relief observed in our study, with all attacks being treated successfully, provides reassurance that the recommended dose of $20 \mathrm{U} / \mathrm{kg}$ for $\mathrm{C} 1-\mathrm{INH}$ concentrate is a reliable therapeutic option for the emergency treatment of laryngeal HAE attacks.

The time period available for the emergency treatment of laryngeal edema lies between the start of the attack and its maximum development. This period was an average of $8.3 \mathrm{~h}$ for untreated attacks in $61 \mathrm{HAE}$ patients and was relatively constant in patients experiencing more than one laryngeal attack [5]. In our study, C1-INH concentrate was mostly administered during the critical time before maximum development of the laryngeal attack, with the infusion being given a median of $2.8 \mathrm{~h}$ after the estimated start of the attack. None of the patients with acute laryngeal attacks treated with C1-INH concentrate in our prospective study, or with the same C1-INH concentrate in the retrospective case-collection study of Bork et al. [5], required emergency procedures such as intubation or tracheotomy, and all patients survived the potentially life-threatening edema. In contrast, intubation or tracheotomy was necessary in $10 \%$ of the 61 patients who were not treated with $\mathrm{C} 1-\mathrm{INH}$ concentrate in the casecollection study and 1 of these patients died of asphyxiation [5]. Only 1 of the 99 patients experiencing laryngeal HAE attacks who were treated with a different $\mathrm{C} 1$-INH concentrate had to undergo tracheotomy despite C1-INH therapy [23].
The safety analyses in our study revealed no signals of concern, concurring with the excellent safety profile of $\mathrm{C} 1$ INH concentrate that has been reported in numerous studies $[2,15,17,22,26,27]$. One concern with the use of plasmaderived products such as C1-INH concentrate is the potential risk for virus transmission to recipients. The manufacture of the highly purified C1-INH concentrate used in our study includes various measures to ensure effective virus and prion reduction, resulting in a high safety margin for this product $[28,29]$. During our study, there was no evidence that the $\mathrm{C} 1-\mathrm{INH}$ treatment was associated with infections of human immunodeficiency virus, hepatitis virus, or parvovirus B19, adding to the experience with more than 400,000 treatments to date with Berinert in patients experiencing acute HAE attacks during which no detectable viral transmission occurred.

\section{Conclusions}

Our results indicate that $\mathrm{C} 1-\mathrm{INH}$ concentrate at the recommended weight-based dose of $20 \mathrm{U} / \mathrm{kg}$ [15] is a highly effective and safe emergency treatment for potentially life-threatening laryngeal attacks in patients with HAE by providing rapid symptom relief in all treated patients. Persistence of efficacy with repeated administration was demonstrated because our study as a whole assessed the use of C1-INH concentrate in the treatment of successive HAE attacks (also for attacks at other body locations). Thus, our findings provide reassurance that $\mathrm{C} 1$ INH concentrate administered at a dose of $20 \mathrm{U} / \mathrm{kg}$ is a reliable, long-term option for the emergency treatment of laryngeal attacks, reducing the risk of asphyxiation should this rare type of HAE attack occur.

Acknowledgements We thank all investigators, subinvestigators, and other members of the I.M.P.A.C.T study group, whose valuable contributions were essential to the success of this study. We thank the members of the Steering Committee (Marco Cicardi [University of Milan, Italy], Henriette Farkas [Semmelweis University, Hungary], and Michael Frank [Duke University, USA]) and the Data Safety Monitoring Board (Konrad Bork [Johannes Gutenberg University Mainz, Germany], Wolfhart Kreuz [J.W. Goethe University, Frankfurt am Main, Germany], and Helmut Sayn [Accovion $\mathrm{GmbH}$, Germany]) for their guidance during the conduct of this study. We are also grateful to Sylvia Herget, Kerstin Jung, Margaret Mitchell, and Xiang Ma (CSL Behring, Germany and USA) for their assistance in the conduct of the study, Silke Kuhl and Silke Jasky-Gamb (Accovion $\mathrm{GmbH}$, Germany) for statistical support, data management, and statistical programming on behalf of CSL Behring $\mathrm{GmbH}$, and Christina Wendel and Douglas Fiebig (Trilogy Writing \& Consulting GmbH, Germany) for medical writing services on behalf of CSL Behring GmbH.

Disclosure of Potential Conflict of Interest Drs. Craig, Wasserman, Levy, Bewtra, Schneider, Packer, and Yang received research support as investigators in this study sponsored by CSL Behring. Dr. Craig reports having also served as a consultant for, and having received research support as, an investigator from, Dyax, Pharming, Shire, and 
ViroPharma. Dr. Wasserman reports having also served as a consultant for Baxter Healthcare, CSL Behring, and Talecris. Drs. Levy, Bewtra, Schneider, Packer, and Yang report no additional sources of funding. Mr. Keinecke is currently an employee of Accovion $\mathrm{GmbH}$, which provides statistical consultancy services to CSL Behring. Dr. Kiessling was employed by CSL Behring during the conduct and reporting of this study.

Open Access This article is distributed under the terms of the Creative Commons Attribution Noncommercial License which permits any noncommercial use, distribution, and reproduction in any medium, provided the original author(s) and source are credited.

\section{References}

1. Bork K. Pasteurized C1 inhibitor concentrate in hereditary angioedema: pharmacology, safety, efficacy and future directions. Expet Rev Clin Immunol. 2008;4:13-20.

2. Cicardi M, Zingale LC, Zanichelli A, et al. The use of plasmaderived $\mathrm{C} 1$ inhibitor in the treatment of hereditary angioedema. Expert Opin Pharmacother. 2007;8:3173-81.

3. Zuraw BL. Hereditary angioedema. $N$ Engl J Med. 2008;359:1027-36.

4. Bork K, Meng G, Staubach P, et al. Hereditary angioedema: new findings concerning symptoms, affected organs, and course. Am J Med. 2006;119:267-74.

5. Bork K, Hardt J, Schicketanz KH, Ressel N. Clinical studies of sudden upper airway obstruction in patients with hereditary angioedema due to $\mathrm{C} 1$ esterase inhibitor deficiency. Arch Intern Med. 2003;163:1229-35.

6. Farkas H, Varga L, Széplaki G, et al. Management of hereditary angioedema in pediatric patients. Pediatrics. 2007;120:e713-22.

7. Bowen T, Cicardi M, Bork K, et al. Hereditary angioedema: a current state-of-the-art review, VII: Canadian Hungarian 2007 International Consensus Algorithm for the Diagnosis, Therapy, and Management of Hereditary Angioedema. Ann Allergy Asthma Immunol. 2008;100(1 Suppl 2):S30-40.

8. Agostoni A, Aygören-Pürsün E, Binkley KE, et al. Hereditary and acquired angioedema: problems and progress: proceedings of the third $\mathrm{C} 1$ esterase inhibitor deficiency workshop and beyond. J Allergy Clin Immunol. 2004;114(3 Suppl):S51-S131.

9. Cicardi M, Bergamaschini L, Marasini B, et al. Hereditary angioedema: an appraisal of 104 cases. Am J Med Sci. 1982;284:2-9.

10. Bork K, Siedlecki K, Bosch S, et al. Asphyxiation by laryngeal edema in patients with hereditary angioedema. Mayo Clin Proc. 2000;75:349-54.

11. Bork K, Barnstedt SE. Laryngeal edema and death from asphyxiation after tooth extraction in four patients with hereditary angioedema. JADA. 2003;134:1088-94.

12. Bork K, Meng G, Staubach P, et al. Treatment with $\mathrm{C} 1$ inhibitor concentrate in abdominal pain attacks of patients with hereditary angioedema. Transfusion. 2005;45:1774-84.

13. Levi M, Choi G, Picavet C, et al. Self-administration of C1inhibitor concentrate in patients with hereditary or acquired angioedema caused by C1-inhibitor deficiency. J Allergy Clin Immunol. 2006;117:904-8.

14. Carugati A, Pappalardo E, Zingale LC, et al. C1-inhibitor deficiency and angioedema. Mol Immunol. 2001;38:161-73.
15. Craig TJ, Levy RJ, Wasserman RL, et al. Efficacy of human C1esterase inhibitor $(\mathrm{C} 1-\mathrm{INH})$ concentrate compared to placebo in patients with acute abdominal or facial HAE attacks. J Allergy Clin Immunol. 2009;124(4):801-8.

16. Bork K, Barnstedt SE. Treatment of 193 episodes of laryngeal edema with $\mathrm{C} 1$ inhibitor concentrate in patients with hereditary angioedema. Arch Intern Med. 2001;161:714-8.

17. Agostoni A, Aygören-Pürsün E, Binkley KE. Hereditary and acquired angioedema: problems and progress: proceedings of the third $\mathrm{C} 1$ esterase inhibitor deficiency workshop and beyond. J Allergy Clin Immunol. 2004;114:S51-S131.

18. Kunschak M, Engl W, Maritsch F, Rosen FS, Eder G, Zerlauth G, et al. A randomized, controlled trial to study the efficacy and safety of $\mathrm{C} 1$ inhibitor concentrate in treating hereditary angioedema. Transfusion. 1998;38(6):540-9.

19. Frank MM. Hereditary angioedema: a current state-of-the-art review, VI: novel therapies for hereditary angioedema. Ann Allergy Asthma Immunol. 2008;100(1 Suppl 2):S23-9.

20. Waytes AT, Rosen FS, Frank MM. Treatment of hereditary angioedema with a vapor-heated $\mathrm{C} 1$ inhibitor concentrate. N Engl J Med. 1996;334(25):1630-4.

21. Zuraw BL. Hereditary angiodema: a current state-of-the-art review, IV: short- and long-term treatment of hereditary angioedema: out with the old and in with the new? Ann Allergy Asthma Immunol. 2008;100(1 Suppl 2):S13-8.

22. Farkas H, Jakab L, Temesszentandrási G, et al. Hereditary angioedema: a decade of human $\mathrm{C} 1$-inhibitor concentrate therapy. J Allergy Clin Immunol. 2007;120(4):941-7.

23. Cicardi M, Zingale L. How do we treat patients with hereditary angioedema. Transfus Apher Sci. 2003;29:221-7.

24. Bork K, Ressel N. Sudden upper airway obstruction in patients with hereditary angioedema. Transfus Apher Sci. 2003;29(3):235-8.

25. Agostoni A, Cicardi M. Hereditary and acquired C1-inhibitor deficiency: biological and clinical characteristics in 235 patients. Medicine. 1992;71:206-15. Baltimore.

26. Gompels MM, Lock RJ, Abinun $\mathrm{M}$, et al. $\mathrm{C} 1$ inhibitor deficiency consensus document. Clin Exp Immunol. 2005; 139: 379-94.

27. Longhurst HJ. Emergency treatment of acute attacks in hereditary angioedema due to $\mathrm{C} 1$ inhibitor deficiency: what is the evidence? Int J Clin Pract. 2005;59:594-9.

28. de Serres J, Gröner A, Lindner J. Safety and efficacy of pasteurized $\mathrm{C} 1$ inhibitor concentrate (Berinert P) in hereditary angioedema: a review. Transfus Apher Sci. 2003;29(3):24754.

29. Gröner A, Nowak T, Schäfer W: An integrated approach to effective pathogen reduction for a plasma-derived $\mathrm{C} 1$ inhibitor concentrate. Kongressausgabe 52. Jahrestagung der Gesellschaft für Thrombose- und Hämostaseforschung e.V. in Wiesbaden. Hämostaseologie 28(1/2) Poster Abstract 2008;P·21-02

\section{Author Contributions' Statement}

PK and HOK conceived the study and designed the trial. TJC, RLW, RJL, AKB, LS, FP, and WY undertook recruitment of patients and managed data, including quality control. HOK performed the statistical analyses. TJC, RLW, RJL, AKB, LS, FP, WY, HOK, and PK provided analysis and interpretation of data and contributed to preparation of the manuscript. TJC takes responsibility for the manuscript as a whole. 